\title{
Selective photocatalytic oxidation of 5-hydroxymethyl-2-furfural in aqueous suspension of polymeric carbon nitride and its adduct with $\mathrm{H}_{2} \mathrm{O}_{2}$ in a solar pilot plant
}

\author{
Marina Ilkaeva1, Igor Krivstov ${ }^{1,2}$, Elisa I. García-López ${ }^{3, *}$, Giuseppe Marcì ${ }^{3}$ Leonardo \\ Palmisano $^{3}$, Ignacio Maldonado ${ }^{4}$, Sixto Malato ${ }^{4}$, José R. García ${ }^{1}$, Eva Díaz ${ }^{5}$, Salvador Ordóñez ${ }^{5}$ \\ ${ }^{1}$ Department of Physical and Analytical and Organic and Inorganic Chemistry, University of \\ Oviedo-CINN, 33006 Oviedo, Spain. \\ ${ }^{2}$ Nanotechnology Education and Research Center, South Ural State University, 454080 \\ Chelyabinsk, Russia \\ 3 "Schiavello-Grillone” Photocatalysis Group. Dipartimento di Energia, Ingegneria \\ dell'informazione e modelli Matematici(DEIM), Università di Palermo, \\ Viale delle Scienze, 90128 Palermo, Italy. \\ ${ }^{4}$ Plataforma Solar de Almería-CIEMAT, Tabernas, Spain. \\ ${ }^{5}$ Department of Chemical and Environmental Engineering, University of Oviedo, \\ 33006 Oviedo, Spain.
}

\section{elisaisabel.garcialopez@unipa.it}

\begin{abstract}
This work reports a study on the reactivity of pristine and $\mathrm{H}_{2} \mathrm{O}_{2}$ treated carbon nitride samples for sunlight photocatalytic selective reactions. The characterization of these materials was reported in a previous paper where the reactivity versus the partial oxidation of 5hydroxymethylfurfural (HMF) was studied by using two different laboratory scale photoreactors; one irradiated by UV lamps and the other one by natural sunlight. In the present study it has been confirmed the effectiveness of these $\mathrm{C}_{3} \mathrm{~N}_{4}$ based materials for the selective partial oxidation of HMF to FDC (2,5-furandicarboxaldehyde) in aqueous medium in a pilot plant photoreactor irradiated by natural solar light. The reactivity results and, in particular, the selectivity to FDC formation have been very encouraging, mostly by considering that the reaction was carried out in water. Moreover, they are comparable with those obtained in the laboratory scale photoreactor irradiated by both UV artificial lamps and natural sunlight. Interestingly, the pristine $\mathrm{C}_{3} \mathrm{~N}_{4}$ sample has shown a higher HMF conversion with respect to that of the $\mathrm{C}_{3} \mathrm{~N}_{4}-\mathrm{H}_{2} \mathrm{O}_{2}$ adduct, but the last one is more selective to the FDC formation. A kinetic study indicates that, the pseudo-
\end{abstract}


first-order rate constant for HMF oxidation is higher in the case of bare photocatalyst and that the equilibrium adsorption constants of $\mathrm{HMF}$ are higher in the case of $\mathrm{C}_{3} \mathrm{~N}_{4}-\mathrm{H}_{2} \mathrm{O}_{2}$ adduct catalyst. Finally, the partial oxidation of two aromatic alcohols, i.e. benzyl alcohol (BA) and 4-methoxy benzyl alcohol (4-MBA) to benzaldehyde (BAL) and 4-methoxy benzaldehyde (4-MBAL), respectively, has also been studied. It has been found that the inductive and delocalization effects as well as the ortho-para orienting ability of the methoxy group with respect to the hydroxyl one affects the conversion of aromatic alcohol and the selectivity towards the corresponding aldehyde.

Keywords: Solar photocatalysis, carbon nitride; selective photocatalytic oxidation; 5hydroxymethyl-2-furfural, 2,5-furandicarboxaldehyde; 4-methoxy-benzyl alcohol; benzyl alcohol

\section{Introduction}

The application of photocatalytic reactions to water and air remediation has been widely studied and thoroughly reviewed [1]. The use of $\mathrm{TiO}_{2}$ as photocatalyst has been demonstrated to be an excellent technology to unselectively degrade many pollutants giving rise to their complete mineralization. However, photocatalysis is also capable to selectively oxidize or reduce a substrate producing a higher value compound. In this new role, photocatalysis appears as an alternative to more conventional synthetic pathways of obtaining value-added chemicals. Heterogeneous photocatalysis offers a greener substitution for many widely applied catalytic oxidations with no toxic by-products and the possible use of solar light as the radiation source. This is an environmentally friendly process carried out at room temperature, using water as the solvent involving cheap and non-toxic oxidants. Many case studies on different organic substrates demonstrating the feasibility of this new approach have been reported until now [2]. The heterogeneous photocatalytic oxidation of alcohols to aldehydes has been carried out in some cases with high yields [3]. Particularly, partial oxidation of aromatic alcohols to aldehydes in the presence of $\mathrm{TiO}_{2}$-based photocatalysts has been successfully performed, although in most of the cases in organic media [4]. $\mathrm{TiO}_{2}$ under $\mathrm{UV}$ irradiation in aqueous medium readily forms hydroxyl radicals, which can unselectively attack organic species until their mineralization to $\mathrm{CO}_{2}$ and $\mathrm{H}_{2} \mathrm{O}$. Consequently, the use of an alternative photocatalytic material with a lower 
oxidant ability can be a good strategy to avoid the complete oxidation of the substrate. In this context, a photocatalyst with appropriate thermodynamic requirements, which has recently attracted attention as a potential candidate to photocatalyse selective partial oxidations is polymeric carbon nitride (PCN). This material is easily prepared by thermal condensation of cyandiamide, melamine, urea or thiourea giving rise to yellow powders showing specific surface areas (SSA) typically in the range of $4-7 \mathrm{~m}^{2} \cdot \mathrm{g}^{-1}$ [5-8]. Its SSA can be increased both by hard or soft templating during the synthesis or by post-synthetic chemical or thermal etching procedures, reaching values up to ca. $300 \mathrm{~m}^{2} \cdot \mathrm{g}^{-1}$ [9]. The electronic structure of the carbon nitride semiconductor allows its successful application in the field of selective photocatalytic oxidation of organic compounds [10], particularly for aromatic alcohols in the presence of molecular oxygen [11,12]. Indeed, the favorable energies of its conduction and valence bands and the absence of hydroxyl groups on the surface, which would promote the direct formation of the unselective $\mathrm{OH}^{\bullet}$ radicals, make this material an optimal candidate to be utilized for selective oxidation of aromatic and non-aromatic alcohols in aqueous medium.

Natural solar radiation can be applied to activate $\mathrm{C}_{3} \mathrm{~N}_{4}$ photocatalyst, because it possesses a band gap in the range of 2.8-3.0 eV, depending on the preparation method. The use of solar light is particularly relevant to develop innovative and economically consistent processes and, at the same time, to move toward sustainable chemistry with a reduced environmental impact.

5-Hydroxymethyl-2-furfural (HMF) is one of the most versatile "biomass platform molecule" produced from abundant and inexpensive lignocellulose-derived glucose [13]. HMF, in its turn, can be transformed into other essential compounds. The presence of alcohol and aldehyde functional groups on the furanic ring gives HMF the possibility to be a precursor of several highvalue chemicals [14]. In particular, HMF can be partially oxidized to 2,5-furandicarboxaldehyde (FDC), 5-hydroxymethyl-2-furancarboxylic acid (HMFA) and 2,5-furandicarboxylic acid (FDCA), all valuable molecules for the polymer industry [15]. Selective oxidation of the alcohol functional group without affecting other functionalities, keeping intact the furan ring, results particularly challenging. The oxidation of the alcohol group forming the corresponding aldehyde (FDC) is an interesting task because this molecule is used as a monomer to synthetize adhesives, binders, foams, antifungal agents, heterocyclic ligands and resins [16]. Generally, the process requires toxic oxidants and generate large amount of hazardous wastes making it environmentally and economically undesirable. These processes have been substituted by 
catalytic oxidation; however, the application of high temperatures, as well as expensive noble metals makes this reaction energy-consuming and environmentally unfriendly [17]. Hu et al. reviewed the different aspects of the synthesis of FDC starting from fructose or HMF [18].

There were several efforts to photocatalytically upgrade HMF, and in most cases FDC was a principal reaction product [19-21], while only with the assistance of a metal-organic cocatalyst FDCA was produced [22]. If the control over the HMF to FDC oxidation selectivity is relatively easily achieved in organic solvents, where hydroxyl radicals are not produced [23], carrying out the same reaction in water is a more complicated task. Low-crystalline and $\mathrm{N}$-doped $\mathrm{TiO}_{2}$ were found to be suitable photocatalysts for this oxidation reaction under UV-light in aqueous medium, however they provided selectivity of only 30-40\% [19,24]. The application of polymeric carbon nitride $(\mathrm{PCN})$ not only allowed to increase this value up to $50 \%$, but also to use efficiently natural sunlight [21]. The following step in the improvement of the selectivity toward FDC production was achieved by $\mathrm{PCN}-\mathrm{H}_{2} \mathrm{O}_{2}$ adduct, which was previously synthesized and characterized [25].

Although the investigation of selective photocatalytic conversion of aromatic alcohols and HMF has advanced significantly in the last two decades, still the research in this field is mainly limited to laboratory studies and only few reports on scaling-up of this process using $\mathrm{TiO}_{2}$ photocatalyst exist [26]. Here, we will demonstrate the applicability of the $\mathrm{PCN}$ and $\mathrm{PCN}-\mathrm{H}_{2} \mathrm{O}_{2}$ adduct photocatalysts for pilot plant scale partial photocatalytic oxidation of HMF to FDC under natural sunlight. Moreover, we will show that the use of the pilot plant compound parabolic collector (CPC) photoreactor for partial photocatalytic oxidation can be extended to syntheses of other valuable chemicals earlier carried out on laboratory scale [27]. For this reason the photooxidation of benzyl alcohol (BA) and 4-methoxybenzyl alcohol (4MBA) to their respective aldehydes, i.e. benzaldehyde (BAL) and 4-methoxybenzaldehyde (4MBAL) will be also discussed (Scheme 1).

The two aromatic alcohols were chosen because they bear different substituent groups which influence, as before reported, their conversion and selectivity [12]. Indeed, the presence of the methoxy group in para position (4-methoxy benzyl alcohol versus benzyl alcohol), increased both reaction rate and selectivity to aldehyde both in the presence of $\mathrm{TiO}_{2}$ [13] and $\mathrm{C}_{3} \mathrm{~N}_{4}$ [12]. Notably, the aldehydes which can be obtained from the photo-oxidation of aromatic alcohols are important intermediates in the chemical, pharmaceutical and agricultural industry. The 
photocatalytic partial oxidation of benzyl alcohol in water to obtain BAL was also reported by using $\mathrm{TiO}_{2}[14,15]$ and $\mathrm{C}_{3} \mathrm{~N}_{4}$ based photocatalysts $[12,16]$. A selectivity to BAL formation of ca. $38-42 \%$ was achieved in studies carried out in the presence of $\mathrm{TiO}_{2}$, while oxidation of 4-MBA to $4-\mathrm{MBAD}$ in water assisted by $\mathrm{TiO}_{2}$ gave rise to a selectivity of $60 \%$ [13] or $72 \%$ [15], depending on the type of $\mathrm{TiO}_{2}$ used.

\section{Experimental}

\subsection{Photocatalyst Preparation and characterization}

Bulk carbon nitride $(\mathrm{BCN})$ was prepared by thermal condensation starting from melamine [21]. The scaled-up preparation of $\mathrm{BCN}$ proceeded by placing $60 \mathrm{~g}$ of melamine in a covered ceramic crucible and by heating it in an oven up to $520{ }^{\circ} \mathrm{C}$ at $2{ }^{\circ} \mathrm{C} \mathrm{min}^{-1}$. The temperature was maintained for $6 \mathrm{~h}$, and after that the system was slowly cooled down giving about $36 \mathrm{~g}$ of $\mathrm{BCN}$. The obtained $\mathrm{BCN}$ consisting of a yellow powder underwent a successive heating in air atmosphere, to obtain thermally etched PCN (TE) with improved physicochemical features $[28,29]$. It was prepared starting from $20 \mathrm{~g}$ of the powdered $\mathrm{BCN}$, which was evenly spread on an aluminium sheet with dimensions $15 \times 22 \mathrm{~cm}$, then heated in air up to $520^{\circ} \mathrm{C}$ at $3{ }^{\circ} \mathrm{C} \mathrm{min}{ }^{-1}$ and kept for $7 \mathrm{~h}$ at this temperature before being cooled down. The thermal etching produced ca. $9 \mathrm{~g}$ of TE, and the procedure was repeated until $200 \mathrm{~g}$ of the thermally etched PCN (TE) was obtained, then TE was powdered and thoroughly mechanically mixed. After that, the pale yellow TE powder was divided into two aliquots, one of them was left as it was, while the other one was treated with $\mathrm{H}_{2} \mathrm{O}_{2}$ according to the procedure reported elsewhere [25,30]. Briefly, $500 \mathrm{~mL}$ of aqueous $\mathrm{H}_{2} \mathrm{O}_{2}$ solution (wt 30\%) containing $100 \mathrm{~g}$ of TE was stirred in an open beaker under heating at $70{ }^{\circ} \mathrm{C}$ to evaporate the liquid. Once dry, the powder was washed with water until the complete absence of $\mathrm{H}_{2} \mathrm{O}_{2}$ in the washing liquid, filtered and dried at $80{ }^{\circ} \mathrm{C}$ for $24 \mathrm{~h}$. The finally obtained dark yellow-to-orange powder was labelled as TEO. The properties of the solids were fully characterized elsewhere [25]. The SSAs of the TE and TEO samples resulted to be 129 and 59 $\mathrm{m}^{2} \mathrm{~g}^{-1}$, respectively.

\subsection{Photocatalytic set-up and procedure}

The photocatalytic experiments were carried out in a pilot plant scale CPC solar photoreactor located at the Plataforma Solar de Almería (Spain). The CPC was not a concentrating one, i.e. the ratio between the sun exposed surface and that of the reactor was approximately equal to 1.1. 
The set-up consisted of a plug flow photoreactor (PFP) in a total recycle loop with a not-reacting stirred tank whose function was providing aeration and sample withdrawing for analyses. The plug flow photoreactor (PFP) was equipped with CPC having five UV-transparent glass tubes (inner diameter $45 \mathrm{~mm}$, outer diameter $50 \mathrm{~mm}$, irradiated length $1460 \mathrm{~mm}$ ) connected in series and placed on a fixed support inclined $37^{\circ}$ (latitude of the PSA) with respect to the horizontal plane and facing South to maximize the daily incidence of solar radiation. The aqueous suspension was continuously fed to the PFP upwards from the not-reacting tank by means of a centrifugal pump. The suspension flow rate, maintained constant for all the runs, was $12 \mathrm{~L} \mathrm{~min}^{-1}$. The Reynolds number value was equal to ca. 5700, indicating a turbulent regime of the flow inside the tubes. The total volume of suspension $\left(\mathrm{V}_{\mathrm{t}}\right)$ charged in the whole system was $15.5 \mathrm{~L}$, whereas the irradiated volume, i.e. the volume of suspension contained in the glass tubes, was 11.6 L. The irradiated volume was $75 \%$ of the total one (dead volume was accounted for to $25 \%$ ). The total irradiated area in the photo-reactor was $1.055 \mathrm{~m}^{2}$ and the suspension slightly heated throughout the experiments, reaching temperatures of ca. $42-45{ }^{\circ} \mathrm{C}$ at the end of the run. A picture of the photoreactor and the reaction set-up is presented in Scheme 2.

Scheme 2

Aqueous solutions of $\operatorname{HMF}(0.5,1,2$ and $4 \mathrm{mM})$ at natural $\mathrm{pH}$ were used for the experiments. For selected runs BA and 4-MBA $(2 \mathrm{mM})$ were also used as the substrates. The photocatalytic activity of the TE and TEO powders was studied together with that of $\mathrm{TiO}_{2}$ Evonik $\mathrm{P} 25$, which was tested for the sake of comparison. The loading of the TE and TEO photocatalysts in the photoreactor ranged between 0.1 and $0.4 \mathrm{~g} \cdot \mathrm{L}^{-1}$. Before starting the photoreactivity runs the aqueous suspension was allowed to recirculate under dark for $20 \mathrm{~min}$ to establish the adsorption/desorption equilibrium between the organic substrate and the solid photocatalyst. Subsequently, the CPC was uncovered and the system was irradiated for $5 \mathrm{~h}$ under sunlight. Samples of the suspension were withdrawn immediately after starting the irradiation and at fixed time intervals $(0.5 \mathrm{~h})$. The samples were withdrawn from the not-reacting tank so that they were representative of the conditions in the inlet. They were filtered through $0.25 \mu \mathrm{m}$ membranes (HA, Millipore) to separate the photocatalyst particles and to analyze the concentration of the substrate and the reaction products. The analyses were carried out by an Agilent 1100 HPLC equipped with a Diode Array detector to identify and to determine concentrations of HMF, FDC 
and 5-formyl-2-furoic acid (FFA). A REZEK ROA Organic acid $\mathrm{H}^{+}$column was used with a mobile phase of aqueous $2.5 \mathrm{mM} \mathrm{H} \mathrm{SO}_{4}$ solution at a flow rate of $1 \mathrm{~mL} \cdot \mathrm{min}^{-1}$. The aromatic molecules, i.e. BA, 4-MBA, BAL and 4-MBAL were analyzed by the same HPLC, but equipped with a Phenomenex KINETEK $5 \mu \mathrm{m}$ C18 instead. The eluent $\left(0.6 \mathrm{~mL} \mathrm{~min}{ }^{-1}\right)$ consisted of a mixture of acetonitrile and $13 \mathrm{mM}$ trifluoroacetic acid (20:80 v:v). Standards purchased from Sigma-Aldrich with a purity $>99 \%$ were used to identify the products and to obtain the calibration curves. Additionally, dissolved organic carbon (DOC) analyses were performed by using a Shimadzu TOC- $V_{C S N}$. The method for monitoring the evolution of $\mathrm{H}_{2} \mathrm{O}_{2}$ during the reaction was based on the formation of a coloured complex between $0.5 \mathrm{~mL}$ of $\mathrm{TiOSO}_{4} 1.5 \%$ (Aldrich) and hydrogen peroxide present in $4.5 \mathrm{~mL}$ of the reacting solution. Then, the concentration of $\mathrm{H}_{2} \mathrm{O}_{2}$ was photometrically determined by measuring the absorbance at $410 \mathrm{~nm}$ using a spectrophotometer UNICAM UV2. The recyclability study was carried out according to the following procedure: At the end of each run the catalyst was left to decant inside the reactor and the supernatant was removed. After that, the reactor was filled with a fresh HMF solution and the subsequent reaction started.

As all of the experiments were carried out under natural solar radiation, it should be considered that the radiation intensity changed throughout the experiments, depending on time and meteorological conditions. Consequently, to properly compare the results, the experimental data were normalized to the amount of energy entering the photoreactor. In the present work this parameter is used instead of the irradiation time to represent the evolution of the concentration of the different substrates during the solar experiments. A Kipp \& Zonen CUV4 (300-400 nm) radiometer, oriented at $37^{\circ}$ South, was applied to automatically measure every minute the power $\left(\mathrm{W} \cdot \mathrm{m}^{-2}\right)$ of the impinging radiation in the 300-400 $\mathrm{nm}$ range. The solar energy accumulated per volume unit throughout the experiment $\left(\mathrm{E}_{\mathrm{n}}\right)$, expressed in $\mathrm{kJ} \cdot \mathrm{L}^{-1}$, was calculated by considering the irradiated area of the photoreactor and the total volume by using Equation (1):

$$
\mathrm{E}_{\mathrm{n}}=\mathrm{E}_{\mathrm{n}-1}+\Delta \mathrm{t}_{\mathrm{n}} \mathrm{W}_{\mathrm{s}}\left(\mathrm{A}_{\mathrm{i}} / \mathrm{V}_{\mathrm{t}}\right)
$$

where $t_{n}$ represents the irradiation time for each sample, $W_{s}$ the average solar radiation measured by the radiometer in each time interval, $\Delta \mathrm{t}_{n}, \mathrm{~V}_{\mathrm{t}}$ is the total reactor volume $(15.5 \mathrm{~L})$ and $\mathrm{A}_{\mathrm{i}}$ the irradiated area of the reactor $\left(1.055 \mathrm{~m}^{2}\right)$. 


\section{Results and discussion}

\subsection{Photocatalytic oxidation of $\mathrm{HMF}$ by using $\mathrm{PCN}$ and $\mathrm{PCN}-\mathrm{H}_{2} \mathrm{O}_{2}$.}

Preliminary adsorption measurements of HMF and FDC under dark in the presence of TE and TEO samples indicated that the extent of adsorption was negligible for both substrates. Blank experiments under solar irradiation of HMF for $5 \mathrm{~h}$ in the absence of photocatalyst showed a decomposition of HMF of ca. $10 \%$ without any selectivity to FDC. On the other hand, ca. $20 \%$ of FDC transformed into FFA under the same experimental conditions, as before observed in the lab scale photoreactor [31]. Nevertheless, it should be considered that the contribution of photolysis in the presence of photocatalyst is always less significant due to the shielding effect of the suspended powder.

The stability of the TEO catalyst under irradiation and dark conditions (in the absence of the substrates) was studied by suspending it in water under vigorous stirring at ca. 50 and $80{ }^{\circ} \mathrm{C}$ for 4 $h$ and measuring the amount of $\mathrm{H}_{2} \mathrm{O}_{2}$ released in water. Only trace quantities of $\mathrm{H}_{2} \mathrm{O}_{2}$ were detected in all cases. Considering that the temperature during the photocatalytic runs under solar irradiation was much lower than $80{ }^{\circ} \mathrm{C}$, this experiment ensured the applicability of the TEO sample for the reaction.

To establish the optimal photocatalyst loading which is necessary to absorb all of the photons impinging on the photoreactor, some photocatalytic tests of HMF oxidation have been carried out in the presence of different amounts $\left(0.1\right.$ to $\left.0.4 \mathrm{~g} \cdot \mathrm{L}^{-1}\right)$ of both the TEO and TE powders, while maintaining $\mathrm{HMF}$ initial concentration at $0.5 \mathrm{mM}$ for all these runs. In Figure 1 (A) the changes of HMF concentration versus the cumulative photonic energy, $E_{n}$, in the presence of different loadings of TEO in the photoreactor are reported. No significant differences can be observed for the experiments carried out in the presence of $0.2,0.33$ or $0.4 \mathrm{~g} \mathrm{~L}^{-1}$ of the photocatalyst, whilst the reaction is noticeably slower for the case of using $0.1 \mathrm{~g} \mathrm{~L}^{-1}$ of TEO. Throughout the photo-oxidation of HMF, FDC is produced together with small amounts of FFA. FDC concentration increases with cumulative energy, as it is shown in Figure 1 (B). The plots of selectivity to FDC versus cumulative energy and HMF conversion at varying TEO loadings are reported in Figures 1 (C) and (D), respectively. It can be noticed that the selectivity to FDC is ca. $90 \%$ up to ca. $20 \%$ of HMF conversion for all the loadings except for $0.1 \mathrm{~g} \mathrm{~L}^{-1}$, but afterwards it 
decreases, although never below ca. $71 \%$ at maximum conversion degree (see Table 1). The obtained values are very high by considering that the reaction is carried out in water and they are very close to those measured by some of us under UV and solar light irradiation using laboratory scale photoreactors [25].

\section{FIGURE 1}

A similar dependency of HMF conversion from the photocatalyst loading is observed for the TE sample. In the presence of $0.1 \mathrm{~g} \mathrm{~L}^{-1}$ of $\mathrm{TE}$, the rate of $\mathrm{HMF}$ oxidation is significantly lower than for the other cases (Fig. 2 (A)). Moreover, the FDC concentration in the reactor (Fig. 2 (B)) and the selectivity toward FDC is also inferior to the values obtained for higher TE loadings (Fig. 2 (C)). The plot of selectivity to FDC formation versus HMF conversion clearly indicates that the TE photocatalyst is less selective than its $\mathrm{PCN}-\mathrm{H}_{2} \mathrm{O}_{2}$ counterpart, the TEO sample, for any given conversion degree (Fig. 2 (D)).

\section{FIGURE 2}

Table 1 shows the HMF conversion and the selectivity to FDC results obtained for different TE and TEO loadings in the photocatalytic runs at $45 \mathrm{~kJ} \cdot \mathrm{L}^{-1}$ of cumulative energy. As far as the TEO catalyst is concerned, the conversion increases with increasing amounts of carbon nitride in the reactor, although it is maintained on the same level for runs carried out with 0.33 and $0.4 \mathrm{~g} \mathrm{~L}^{-1}$ of $\mathrm{PCN}-\mathrm{H}_{2} \mathrm{O}_{2}$. In addition, the selectivity to FDC increases reaching a plateau for photocatalyst loading higher or equal to $0.33 \mathrm{~g} \mathrm{~L}^{-1}$ (71 \% at $47 \%$ of $\mathrm{HMF}$ conversion, after $45 \mathrm{~kJ} \cdot \mathrm{L}^{-1}$ of cumulative energy), indicating that this photocatalyst concentration corresponds to an optimal value. Also, in the case of TE, the optimal catalyst loading is $0.33 \mathrm{~g} \mathrm{~L}^{-1}$, but the photoactivity of this material is much higher with respect to that of TEO, reaching the HMF conversion of $77 \%$ under the same experimental conditions, although demonstrating lower selectivity to FDC of only $28 \%$ (Table 1). Interestingly, also in this case the reactivity of TE is close to that measured by some of us in a solar and in an UV-irradiated laboratory-scale photoreactors [25]. It is worth noticing that $\mathrm{PCN}$ and $\mathrm{PCN}-\mathrm{H}_{2} \mathrm{O}_{2}$ possess low capability to mineralize the substrate (Table 1). Notably, an experiment carried out under the same experimental conditions but in the presence 
of $0.2 \mathrm{~g} \mathrm{~L}^{-1}$ of $\mathrm{TiO}_{2}$ Evonik P25 (this amount was the optimum for P25 as was previously established for the same photoreactor [32,33] showed the total mineralization of HMF at ca. 25 $\mathrm{kJ} \cdot \mathrm{L}^{-1}$, being the selectivity versus FDC virtually zero at any cumulative energy value of the run.

\section{TABLE 1}

A perusal of the data reported in Figs. 1 (A), (B) and 2 (A), (B) indicates that a straight-line fits both the initial variation of HMF concentration and the FDC formation, although the latter deviates from this trend at high cumulative energy values due to its transformation into FFA.

Table 2 reports the values of the initial reaction rates of HMF photo-oxidation and FDC formation at the varying loadings of TEO and TE. These values are also shown in Figures 3(A) and (B) for the TEO and TE photocatalysts, respectively. From Table 2 and Fig. 3 it can be observed that the initial HMF disappearance rate is always nearly the same as the initial formation rate of FDC in all of the runs, but only in the case of the TEO catalyst. This finding suggests that in the presence of TEO the oxidation of HMF proceeds by one-step reaction giving rise to FDC. On the contrary, the initial reaction rate of HMF is nearly twice as higher than the initial FDC formation rate in the case of the TE photocatalyst, indicating on the presence of parallel reactions. One can suppose that the formation of open-chain aliphatic compounds might take place. As it is evidenced from the HPLC study, several intermediate compounds, other than FDC and FFA, supposedly attributed to the class of carboxylic acids, are produced while HMF is being oxidized with the assistance of TE. However, the formation of FFA is not likely to be attributed to any parallel pathway of HMF photo-oxidation, since its production correlates well with the FDC concentration in the reactor. It is highly probable that FFA is the principal product of the photolysis of FDC, as previously was suggested [21].

\section{TABLE 2}

\section{FIGURE 3}

Once the optimal photocatalyst loading equal to $0.33 \mathrm{~g} \mathrm{~L}^{-1}$ for both of the used materials had been established, a systematic investigation of the influence of the initial HMF concentration on the reaction rate and on the selectivity to FDC was carried out. Figures 4 and 5 report the concentrations of HMF and FDC versus the incident cumulative photonic energy and the 
selectivity to FDC versus both the cumulative energy and the HMF conversion for the runs carried out applying different initial HMF concentrations in the presence of the TEO and TE photocatalysts, respectively.

As a general trend, it is observed that the increase of the initial HMF concentration favours the enhancement of the reaction rate of the HMF disappearance as well as that of the FDC formation (Figs. 4 (A), (B) and Figs. 5 (A), (B)). As far as the selectivity to FDC is concerned, it is seen that the TEO sample is still the most selective of the two (Figs. 4 (C), (D) and Figs. 5 (C), (D)). Notably, during the reaction the selectivity to FDC decreases with increasing the HMF conversion, and this is particularly evident in the case of the TE photocatalyst (Fig. 5). The selectivity to FDC is found to be lower for the highest HMF initial concentration at $45 \mathrm{~kJ} \mathrm{~L}^{-1}$ of cumulative energy (Table 3). The most plausible explanation to this fact might be the presence in the reactor of high concentration of hydrogen peroxide produced according to the mechanism represented in Scheme 3. The concentration of hydrogen peroxide formed throughout the photoreactions carried out in the presence of TEO and TE, respectively, is reported in Figures 6 (A) and (B). High $\mathrm{H}_{2} \mathrm{O}_{2}$ content in the irradiated suspension can promote its reaction with photogenerated electrons leading to the production of highly oxidative ${ }^{\circ} \mathrm{OH}$ radicals, which are responsible for the ring-opening reaction as well as for the complete substrate mineralization (Table 3).

\section{FIGURE 4}

FIGURE 5

\section{FIGURE 6}

The initial reaction rates of HMF disappearance and FDC formation calculated from the reaction data are represented on Figure 7 and summarized in Table 4 for the runs carried out in the presence of the TEO and TE catalysts. The initial reaction rate both for HMF oxidation and for FDC formation increases by increasing the initial HMF concentration. For TEO catalyst the value of the initial reaction rate of HMF conversion is very close to that of FDC formation only for the runs with the lowest HMF initial concentration (0.5 and $1 \mathrm{mM})$. On the contrary, for higher initial HMF concentrations and for the TE catalyst in all the cases, the initial reaction rate 
of HMF conversion is higher than that of FDC formation, indicating the occurrence of parallel oxidation processes from the early reaction stages.

Table 4 also reports the initial formation rate of $\mathrm{H}_{2} \mathrm{O}_{2}$ in the presence of both photocatalysts. Interestingly, in the case of using the TEO photocatalyst, for which the HMF to FDC oxidation is the primary reaction and the only one at low conversion rates of $\mathrm{HMF}$, the initial formation rates of FDC and $\mathrm{H}_{2} \mathrm{O}_{2}$ nearly coincide (Table 4). On the contrary, for the TE photocatalyst the initial formation rate of $\mathrm{H}_{2} \mathrm{O}_{2}$ is far superior to that of FDC (Table 4). It has been confirmed that, despite the TEO photocatalyst is prepared in the presence of $\mathrm{H}_{2} \mathrm{O}_{2}$, it does not release $\mathrm{H}_{2} \mathrm{O}_{2}$ in water under irradiation, nor if heated. Moreover, the material is stable throughout the reaction runs, as above discussed. Another possible way of $\mathrm{H}_{2} \mathrm{O}_{2}$ photocatalytic production is through the series of consecutive reduction reaction from water and oxygen [31]. However, no $\mathrm{H}_{2} \mathrm{O}_{2}$ has been detected in the irradiated aqueous suspensions of any of the PCN catalysts in the absence of organic substrate. $\mathrm{H}_{2} \mathrm{O}_{2}$ detected in the reaction suspensions appears in the result of organic substrate oxidation.

TABLE 4

FIGURE 7

The initial formation rates of FDC and $\mathrm{H}_{2} \mathrm{O}_{2}$ are very close when TEO catalyst is used, indicating the simultaneous formation of the two species during the early stages of the reaction, as it was observed as well by Shiraishi et al. [34]. Consequently, the mechanism depicted on Scheme 3 can be hypothesized. The superoxide radical, $\mathrm{O}_{2}{ }^{--}$, that is the most abundant and the most likely oxidant species present in the reaction environment (see section 3.2 kinetic aspects), attacks the substrate forming $\mathrm{HMF}^{-}$and a hydro-peroxide radical, which is transformed into $\mathrm{H}_{2} \mathrm{O}_{2}$ by abstracting a hydrogen atom from $\mathrm{HMF}^{-}$giving rise, in turn, to the formation of a $\mathrm{HMF}^{\bullet-}$ radical species, in a similar way to that was previously proposed in [35]. This last species can be oxidized by the hole forming FDC. This mechanism shows that for each mole of FDC produced, one mole of $\mathrm{H}_{2} \mathrm{O}_{2}$ is also obtained. In the presence of $\mathrm{TE}$, the initial formation rate of $\mathrm{H}_{2} \mathrm{O}_{2}$ is higher than that of FDC. In this case, as above discussed, the occurrence of at least two 
parallel reactions is evident, consequently a similar mechanism, in which $\mathrm{H}_{2} \mathrm{O}_{2}$ is formed also during the other parallel reaction, can also be hypothesized.

In order to test the reusability of the most selective TEO catalyst, three consecutive runs were carried out. The decrease of both reactivity and selectivity is accounted for ca. $10 \%$ after each cycle. This behaviour is very different with respect to that observed in laboratory scale reactor, in which the catalysts maintained the same HMF conversion degree and the same selectivity to FDC during all the runs [21,25]. Probably, the impossibility to wash the photocatalysts and its loss after the runs in the solar pilot plant photoreactor are responsible for the lowered efficiency of $\mathrm{PCN}-\mathrm{H}_{2} \mathrm{O}_{2}$.

\section{Scheme 3}

\subsection{Kinetic aspects}

The inability of carbon nitride to produce ${ }^{\circ} \mathrm{OH}$ radicals by direct interaction of electron-holes with water, does not favour the degradation of FDC, making HMF partial oxidation the prevailing process. In previous studies [21], the presence of molecular oxygen (aerobic atmosphere) was necessary to obtain FDC; indeed, the superoxide radical formed via reduction of $\mathrm{O}_{2}$ by photo generated electrons was established to be the main reactive species responsible for the HMF partial oxidation to FDC in water.

The rate determining step of the photo-oxidation process is hypothesised to be the reaction between $\mathrm{O}_{2}{ }^{\cdot-}$ and $\mathrm{HMF}$ on the catalyst surface. As the adsorbed oxygen acts as an electron trap thus hindering the electron-hole recombination, the $\mathrm{O}_{2}{ }^{-}$radicals concentration depends on the fractional sites coverage by $\mathrm{O}_{2}$. Two different types of sites are hypothesised to exist on the catalyst surface. The first ones can adsorb HMF while the second ones adsorb oxygen. In this hypothesis the initial reaction rate $(r)$ for second-order surface oxidation of HMF can be written in terms of Langmuir-Hinshelwood kinetics as:

$r=k^{\prime \prime} \theta_{\text {oxygen }} \theta_{H M F}$

eq. 1 
in which $k^{\prime \prime}$ is the surface second-order rate constant, and $\theta_{\text {oxygen }}$ and $\theta_{H M F}$ are the fractional sites coverages by oxygen and HMF, respectively. The fractional sites coverages by oxygen and HMF are given by:

$\theta_{\text {oxygen }}=\frac{K_{O x} c_{O x}}{1+K_{O x} c_{O x}}$ eq. 2

$\theta_{H M F}=\frac{K_{H M F} c_{H M F}}{1+K_{H M F} c_{H M F}}$ eq. 3

in which $K_{O X}$ and $K_{H M F}$ are the equilibrium adsorption constants of oxygen and HMF, respectively, and $c_{O x}$, and $c_{H M F}$, the oxygen and $\mathrm{HMF}$ concentrations in the aqueous phase. As all the experiments were performed in an open batch reactor in contact with atmospheric oxygen, it may be assumed that the $\theta_{\text {oxygen }}$ term is constant throughout the HMF photo-oxidation. eq. 1 can therefore be written as:

$r=k^{\prime} \theta_{H M F}$ eq. 4

in which $k^{\prime}$ is the surface pseudo-first-order rate constant and it is equal to $k^{\prime \prime} \theta_{\text {oxygen }}$. By substituting eq. 3 into eq. 4 , the following equation is obtained:

$r=k^{\prime} \frac{K_{H M F} c_{H M F}}{1+K_{H M F} c_{H M F}}$ eq. 5

and consequently:

$\frac{1}{r}=\frac{1}{k^{\prime}} \frac{1+K_{H M F} c_{H M F}}{K_{H M F} c_{H M F}}=\frac{1}{k^{\prime}}+\frac{1}{k^{\prime} K_{H M F}} \frac{1}{c_{H M F}}$ eq. 6

The experimental data of the reciprocal of the initial HMF concentration $\left(\frac{1}{c_{H M F}}\right)$ and the corresponding reciprocal of the initial reaction rate $\left(\frac{1}{r}\right)$ in the Cartesian coordinates plane $\left(\frac{1}{c_{H M F}}, \frac{1}{r}\right)$ for runs carried out in the presence of the TEO and TE catalysts, respectively, are 
shown in Figures 8 (A) and (B). In that plane, equation 6 represents a straight line whose slope is $\frac{1}{k^{\prime} K_{H M F}}$ and whose intercept, with the $\frac{1}{r}$ axis, is $\frac{1}{k^{\prime}}$ (Fig. 8).

By applying a least-square best fitting procedure to these data (dotted lines in Figure 8 represent the interpolating equation), it is possible to calculate $k^{\prime}$ and $K_{H M F}$ values for the two catalysts used. These values are reported in Table 5. The fitting of the experimental data is very good, particularly in the case of the TE photocatalyst, validating the kinetic model. The results indicate that, the surface pseudo-first-order rate constant of HMF degradation is higher in the case of the TE photocatalyst but that the equilibrium adsorption constants of HMF is higher in the case of the TEO catalyst. This last finding suggests that the saturation of the catalytic sites can be reached more easily in the case of TEO, probably because, as hypothesized in the previous work [25], this catalyst present a lower number of active sites with respect to the TE photocatalyst, which is also expected in a view of its lower SSA.

\section{FIGURE 8, TABLE 5}

\subsection{Photocatalytic oxidation of BA and 4MBA by using TE and TEO}

Figure 9 reports the obtained results. It can be observed that, by using both photocatalysts, the conversion of 4MBA was faster with respect to that of $\mathrm{BA}$ as well as the formation of the corresponding aldehydes how it is shown in Figures 9 (A) and (B), respectively. Moreover, the reactivity of the TE material is higher with respect to that of TEO. The selectivity to the corresponding aldehydes, reported in Figure 9 (C), appears higher for BA than for 4MBA. However, if compared at the same conversion percentage of the alcohol (see Figure 9(D)) it is evident that: i) up to ca. $20 \%$ of alcohol conversion the selectivity to both aldehydes is ca. 100 $\%$; ii) for higher conversion degrees the selectivity is higher for 4MBAL (this last comparison was possible only by using TE catalyst because the maximum conversion of BA in the presence of TEO is only of $19 \%$ ). The higher conversion of 4MBA with respect to BA and selectivity to 4MBAL with respect to BAL were observed in the previous investigation using the $\mathrm{C}_{3} \mathrm{~N}_{4}$ based photocatalysts [27]. Although, the presence, in 4MBA, of a methoxy group in para position with respect to the alcoholic one determines both an inductive and a delocalization effect that hinder the oxidant attack which can cause the substrate mineralization [27,36], since methoxy group is 
also ortho-para orienting, it induces the oxidant attack to the alcoholic group, thus favouring the aldehyde formation. By comparing this results with those obtained for HMF partial oxidation under the same conditions (see Table 6) it can be seen that: i) the conversion of HMF is intermediate to that of the aromatic alcohols and ii) the selectivity to the corresponding aldehydes is higher for the aromatic compounds. The higher selectivity to aromatic aldehyde with respect to FDC is not surprising, because it is well known that furanic ring of HMF is much less stable than the aromatic one, and, consequently, during the oxidative attack its breakage is most probable. This last finding can also explain why the conversion of HMF is higher with respect to that of BA in the photocatalytic tests.

FIGURE 9

\section{Acknowledgments}

Authors wish to thank CIEMAT-PSA and European Commission for the funding in the framework of the EU-DG RTD's project: SFERA II contract $\mathrm{n}^{\circ} 312643$. University of Oviedo acknowledge the financial support from Spanish MINECO (MAT2016-78155-C2-1-R). IK thanks for the support the Ministry of Education and Science of the Russian Federation (grant no. 4.9722.2017/8.9). 
Table 1. Photoreactivity results for the $\mathrm{TEO}, \mathrm{TE}$ and $\mathrm{TiO}_{2}$ Evonik $\mathrm{P} 25$ (for the sake of comparison) photocatalyts with $0.5 \mathrm{mM} \mathrm{HMF}$ initial concentration. HMF conversion (X $\mathrm{XMF}_{\mathrm{H}}$, selectivity towards FDC (SFC), and dissolved organic carbon (DOC) decrease after $45 \mathrm{~kJ} \cdot \mathrm{L}^{-1}$ (25 $\mathrm{kJ} \cdot \mathrm{L}^{-1}$ in the case of P25) of cumulative energy entering the photoreactor.

\begin{tabular}{|c|c|c|c|c|c|c|}
\hline \multirow{2}{*}{$\begin{array}{c}\text { Photocatalyst loading } \\
\left.\text { [g. } \mathrm{L}^{-1}\right]\end{array}$} & \multicolumn{2}{|c|}{$\mathrm{X}_{\mathrm{HMF}}[\%]$} & \multicolumn{2}{|c|}{$\mathrm{S}_{\mathrm{FDC}}[\%]$} & \multicolumn{2}{|c|}{$\mathrm{DOC}[\%]$} \\
\hline & TEO & $\mathrm{TE}$ & TEO & $\mathrm{TE}$ & TEO & $\mathrm{TE}$ \\
\hline 0.1 & 34 & 62 & 51 & 25 & 5 & 8 \\
\hline 0.2 & 41 & 77 & 65 & 28 & 2 & 11 \\
\hline 0.33 & 47 & 77 & 71 & 28 & 4 & 13 \\
\hline 0.4 & 47 & - & 71 & - & 3 & - \\
\hline & \multicolumn{2}{|c|}{$\mathrm{P} 25$} & \multicolumn{2}{|c|}{$\mathrm{P} 25$} & \multicolumn{2}{|c|}{$\mathrm{P} 25$} \\
\hline 0.2 & \multicolumn{2}{|c|}{100} & \multicolumn{2}{|c|}{0} & \multicolumn{2}{|c|}{100} \\
\hline
\end{tabular}


Table 2. HMF initial oxidation rate $\left(\mathrm{r}_{\mathrm{HMF}}\right)$ and FDC initial formation rate $\left(\mathrm{r}_{\mathrm{FDC}}\right)$ in the presence of the TEO and TE as photocatalysts with HMF $0.5 \mathrm{mM}$ initial concentration.

\begin{tabular}{|c|c|c|c|c|}
\hline $\begin{array}{c}\text { Photocatalyst } \\
\text { loading } \\
{\left[\mathrm{g} \cdot \mathrm{L}^{-1}\right]}\end{array}$ & \multicolumn{2}{|c|}{ rHMF } & \multicolumn{2}{c|}{ rFDC } \\
\hline $\left.\mathrm{mmol} \cdot \mathrm{kJ}^{-1}\right] 10^{3}$ & \multicolumn{2}{c|}{} \\
\hline & TEO & TE & TEO $\left.\cdot \mathrm{kJ}^{-1}\right] 10^{3}$ & TE \\
\hline 0.1 & 4.90 & 8.80 & 5.10 & 4.10 \\
\hline 0.2 & 6.40 & 11.0 & 6.10 & 6.00 \\
\hline 033 & 7.30 & 10.9 & 6.60 & 6.00 \\
\hline 0.4 & 7.10 & - & 6.40 & - \\
\hline & & & & \\
\hline
\end{tabular}


Table 3. Photoreactivity results in the presence of $0.33 \mathrm{~g} \mathrm{~L}^{-1}$ of the TEO and TE photocatalyts at different HMF initial concentrations: HMF conversion $\left(\mathrm{X}_{\mathrm{HMF}}\right)$, selectivity to FDC ( $\mathrm{S}_{\mathrm{FDC}}$ ) and dissolved organic carbon (TOC) decrease after $45 \mathrm{~kJ} \cdot \mathrm{L}^{-1}$ of cumulative energy.

\begin{tabular}{|c|c|c|c|c|c|c|}
\hline $\begin{array}{c}\text { [HMF] } \\
{[\mathrm{mM}]}\end{array}$ & \multicolumn{2}{|c|}{$\mathrm{X}_{\mathrm{HMF}}[\%]$} & \multicolumn{2}{c|}{$\mathrm{S}_{\mathrm{FDC}}[\%]$} & \multicolumn{2}{c|}{ DOC [\%] } \\
\hline & TEO & TE & TEO & TE & TEO & TE \\
\hline 0.5 & 47 & 77 & 71 & 28 & 4 & 13 \\
\hline 1 & 26 & 69 & 70 & 24 & 2 & 11 \\
\hline 2 & 26 & 57 & 61 & 22 & 2 & 9 \\
\hline 4 & 20 & 49 & 45 & 21 & 2 & 6 \\
\hline
\end{tabular}


Table 4. Photoreactivity results in the presence of $0.33 \mathrm{~g} \mathrm{~L}^{-1}$ of the TEO and TE photocatalyts at different HMF initial concentrations: HMF initial oxidation rate $\left(\mathrm{r}_{\mathrm{HMF}}\right)$, FDC initial formation rate ( $\mathrm{r} F \mathrm{PC})$ and initial $\mathrm{H}_{2} \mathrm{O}_{2}$ formation rate ( $\left.\mathrm{r}_{2} \mathrm{O} 2\right)$.

\begin{tabular}{|c|c|c|c|c|c|c|}
\hline $\begin{array}{c}{[\mathrm{HMF}]} \\
{[\mathrm{mM}]}\end{array}$ & \multicolumn{2}{|c|}{ rHMF } & \multicolumn{2}{c|}{ rFDC } & \multicolumn{2}{c|}{ r 2 2O2 } \\
& \multicolumn{2}{|c|}{$\left[\mathrm{mmol} \cdot \mathrm{kJ}^{-1}\right] 10^{3}$} & \multicolumn{2}{c|}{$\left[\mathrm{mmol} \cdot \mathrm{kJ}^{-1}\right]$} & $10^{3}$ & \multicolumn{2}{c|}{$\left[\mathrm{mmol} \cdot \mathrm{kJ}^{-1}\right] 10^{3}$} \\
\hline & TEO & TE & TEO & TE & TEO & TE \\
\hline 0.5 & 7.30 & 10.9 & 6.60 & 6.00 & 6.50 & 10.4 \\
\hline 1 & 8.70 & 20.6 & 6.90 & 10.0 & 6.90 & 13.9 \\
\hline 2 & 15.0 & 29.9 & 11.9 & 12.1 & 12.0 & 18.7 \\
\hline 4 & 20.0 & 51.0 & 13.6 & 19.3 & 12.9 & 27.6 \\
\hline
\end{tabular}

Table 5. Surface pseudo-first-order rate constant $\left(k^{\prime}\right)$ and HMF equilibrium adsorption constants $\left(K_{H M F}\right)$ calculated for TEO and TE as photocatalyts

\begin{tabular}{|c|c|c|}
\cline { 2 - 3 } \multicolumn{1}{c|}{} & TEO & TE \\
\hline$k^{\prime} \cdot 10^{6}\left(\mathrm{~mol}^{1} \mathrm{~kJ}^{-1}\right)$ & 22 & 95 \\
\hline$K_{H M F}\left(\mathrm{M}^{-1}\right)$ & 925 & 265 \\
\hline
\end{tabular}


Table 6. Photoreactivity results in the presence of $0.33 \mathrm{~g} \mathrm{~L}^{-1}$ of the TEO and TE photocatalyts for the three different substrates at $2 \mathrm{mM}$ initial concentration: Initial alcohol oxidation rate ( $\mathrm{r}_{\text {alcohol}}$ ), initial aldehyde formation rate ( $\mathrm{r}_{\text {aldehyde) }}$, alcohol conversion $(\mathrm{X})$ and selectivity to the aldehyde (S) after $45 \mathrm{~kJ} \cdot \mathrm{L}^{-1}$ of cumulative energy.

\begin{tabular}{|c|c|c|c|c|c|c|}
\cline { 2 - 7 } \multicolumn{1}{c|}{} & \multicolumn{3}{c|}{ TE } & \multicolumn{3}{c|}{ TEO } \\
\cline { 2 - 7 } \multicolumn{1}{c|}{} & 4-MBA & BA & HMF & 4-MBA & BA & HMF \\
\hline $\mathrm{r}_{\text {alcohol }}$ & 85.8 & 16.6 & 29.9 & 29.1 & 15.0 & 15.0 \\
\hline mmol.kJ-1] $10^{3}$ & & & & & & \\
\hline raldehyde $_{\left[\mathrm{mmol} \cdot \mathrm{kJ}^{-1}\right] 10^{3}}$ & 84.0 & 16.6 & 12.1 & 28.9 & 15.0 & 11.9 \\
\hline $\mathrm{X}[\%]$ & 81 & 28 & 57 & 48 & 19 & 26 \\
\hline $\mathrm{S}[\%]$ & 67 & 83 & 22 & 81 & 100 & 61 \\
\hline
\end{tabular}




\section{CAPTION FOR FIGURES AND SCHEMES}

Scheme 1. Structure of the alcohols and their corresponding aldehyde investigated.

Scheme 2. Pilot plant photoreactor (A) and set-up (B).

Scheme 3. Hypothesized mechanism of $\mathrm{FDC}$ and $\mathrm{H}_{2} \mathrm{O}_{2}$ formation during the course of the reaction.

Figure 1. HMF concentration (A), FDC concentration (B), selectivity to FDC versus cumulative energy (C), and selectivity to FDC versus HMF conversion (D) in the presence of different loadings of TEO: $0.1 \mathrm{~g} \mathrm{~L}^{-1}(\boldsymbol{\Delta}) ; 0.2 \mathrm{~g} \mathrm{~L}^{-1}(\diamond) ; 0.33 \mathrm{~g} \mathrm{~L}^{-1}(\square)$ and $4 \mathrm{~g} \mathrm{~L}^{-1}(\bullet)$. Initial HMF concentration: $0.5 \mathrm{mM}$.

Figure 2. HMF concentration (A), FDC concentration (B), selectivity to FDC versus cumulative energy (C), and selectivity to FDC versus HMF conversion (D) in the presence of different loadings of TE: $0.1 \mathrm{~g} \mathrm{~L}^{-1}(\bullet) ; 0.2 \mathrm{~g} \mathrm{~L}^{-1}(\Delta)$; $0.33 \mathrm{~g} \mathrm{~L}^{-1}(\diamond)$. Initial HMF concentration: $0.5 \mathrm{mM}$.

Figure 3. Initial HMF degradation rate ( $\Delta$ ) and initial FDC formation rate ( $\square$ ) versus the loading of the TEO (A) and TE (B) photocatalysts. Initial HMF concentration: $0.5 \mathrm{mM}$.

Figure 4. HMF concentration (A), FDC concentration (B), selectivity to FDC versus cumulative energy (C) and selectivity to FDC versus HMF conversion (D) at different initial concentrations of HMF: $0.5 \mathrm{mM}(\diamond) ; 1 \mathrm{mM}(\square) ; 2 \mathrm{mM}(\Delta)$ and $4 \mathrm{mM}(\bullet)$. Loading of TEO: $0.33 \mathrm{~g} \mathrm{~L}^{-1}$. 
Figure 5. HMF concentration (A), FDC concentration (B), selectivity to FDC versus cumulative energy (C) and selectivity to FDC versus HMF conversion (D) at different initial concentrations of HMF: $0.5 \mathrm{mM}(\diamond) ; 1 \mathrm{mM}(\square) ; 2 \mathrm{mM}(\Delta)$ and $4 \mathrm{mM}(\bigcirc)$. Loading of TE $0.33 \mathrm{~g} \mathrm{~L}^{-1}$.

Figure 6. $\mathrm{H}_{2} \mathrm{O}_{2}$ concentration versus cumulative energy for different initial concentrations of HMF: $0.5 \mathrm{mM}(\diamond) ; 1 \mathrm{mM}(\square) ; 2 \mathrm{mM}(\Delta)$ and $4 \mathrm{mM}(\bigcirc)$ for runs carried out in the presence of TEO (A) and TE (B) photocataly. Loading of catalyst $0.33 \mathrm{~g} \mathrm{~L}^{-1}$.

Figure 7. Initial HMF degradation rate $(\boldsymbol{\Delta})$ and initial FDC formation rate ( $\square$ ) versus HMF concentration in the presence of TEO (A) and TE (B) photocatalysts. Catalyst loading of $0.33 \mathrm{~g} \mathrm{~L}^{-1}$.

Figure 8. Reciprocal of the initial HMF concentration $\left(\frac{1}{c_{H M F}}\right)$ vs. the corresponding reciprocal of the initial reaction rate $\left(\frac{1}{r}\right)$ for runs carried out in the presence of the TEO (A) and TE (B) photocatalysts, respectively. The dotted lines represent the interpolating equations obtained by applying the least-square best fitting procedure to the experimental data.

Figure 9. BA (squares) and 4-MBA (circles) concentrations (A); BAL (squares) and 4-MBAL (circles) concentrations (B); selectivity to BAL (squares) and 4-MBAL (circles) versus cumulative energy (C) and selectivity to BAL (squares) and 4-MBAL (circles) versus BA and 4MBA conversion, respectively (D) in the presence of $0.33 \mathrm{~g} \mathrm{~L}^{-1}$ of TE (full symbols) and TEO (void symbols). Initial alcohol concentration $2 \mathrm{mM}$. 


\section{REFERENCES}

[1] D. Spasiano, R. Marotta, S. Malato, P. Fernandez-Ibáñez, I. Di Somma, Appl. Catal. B Environ., 170 (2015) 90-123.

[2] X. Lang, X. Chen, J. Zhao, Chem. Soc. Rev., 46 (2014) 473-486.

[3] L. Palmisano, V. Augugliaro, M. Bellardita, A. Di Paola, E. García-López, V. Loddo, G. Marcì, G. Palmisano, S. Yurkadal, ChemSusChem, 4 (2011) 1431-1438.

[4] J.C. Colmenares, W. Ouyang, M. Ojeda, E. Kuna, O. Chernyayeva, D. Lisovytskiy, S. De, R. Luque, A. M. Balu, Appl. Catal. B. 183 (2016) 107-112.

[5] X. Wang, K. Maeda, A. Thomas, K. Takanabe, G. Xin, J.M. Carlsson, K. Domen, M. Antonietti, Nature Mater. 8 (2009) 76-80.

[6] Y. Wang, Y. Di, M. Antonietti, H. Li, X. Chen, X. Wang, Chem. Mater. 22 (2010) 51195121.

[7] K. Wang, Q. Li, B. Liu, B. Cheng, W. Ho, J. Yu, Appl. Catal. B. 176-177 (2015) 44-52.

[8] Y.W. Zhang, J.H. Liu, G. Wu, W. Chen, Nanoscale, 4 (2012) 5300-5303.

[9] Z. Yang, Y. Zhang, Z. Schnepp, J. Mater. Chem. A. 3 (2015) 14081-14092.

[10] X. Wang, S. Blechert, M. Antonietti, ACS Catal., 2 (2012) 1596-1606.

[11] S. Verma, R.B.N Baig, M.N. Nadagouda, R.S. Varma, ACS Sustain. Chem. Eng. 4 (2016) 1094-1098.

[12] B. Long, Z. Ding and X. Wang, ChemSusChem, 6 (2013) 2074-2078.

[13] J. Wang, J. Xi, Q. Xia, X. Liu, Y. Wang, Sci. China Chem. 60 (2017) 870-886.

[14] M.J. Climent, A. Corma, S. Iborra, Green Chem., 16 (2014) 516-547.

[15] L. Hu, L. Linc, Z. Wu, S. Zhou, S. Liu, Renew. Sus. Eng. Rev. 74 (2017) 230-257.

[16] M. Chatterjee, T. Ishizaka, A. Chatterjee, H. Kawanami, Green Chem., 19 (2017) 13151326.

[17] A.S. Amarasekara, D. Green, E. McMillan, Catal.Commun. 9 (2008) 286-288.

[18] L. Hu, G. Zhao, W. Hao, X. Tang, Y. Sun, L. Lin, S. Liu, RSC Adv. 2 (2012) 11184-11206.

[19] S. Yurdakal, B.S. Tek, O. Alagoz, V. Augugliaro, V. Loddo, G. Palmisano, L. Palmisano, ACS Sust. Chem. Eng. 1 (2013) 456-461.

[20] L. Ozcan, P. Yalcin, O. Alagoz, S. Yurdakal, Catal. Today, 281 (2017) 213. 
[21] I. Krivstov, E.I. García-López, G. Marcì, L. Palmisano, Z. Amghouz, J.R. García, S. Ordóñez, E. Díaz, Appl. Catal. B, 204 (2017) 430-439.

[22] S. Xu, P. Zhou, Z. Zhang, C. Yang, B. Zhang, K. Deng, S. Bottle, H. Zhu, J. Am. Chem. Soc., 139 (2017) 14775-14782.

[23] H. Zhang, Q. Wu, C. Guo, Y. Wu, T. Wu, ACS Sus. Chem. Eng., 5 (2017) 3517-3523.

[24] I. Krivtsov, M. Ilkaeva, E. Salas-Colera, Z. Amghouz, J.R. García, E. Díaz, S. Ordóñez, S. Villar-Rodil, J. Phys. Chem. C, 121 (2017) 6770-6780.

[25] Green Chem submitted.

[26] D. Spasiano, L.P.P. Rodriquez, J.C. Olleros, S. Malato, R. Marotta, R. Andreozzi, Appl. Catal. B: Environ. 136-137 (2013) 56-63.

[27] M. Bellardita, E.I. García-López, G. Marcì, I. Krivtsov, J.R. García, L. Palmisano, Appl. Catal. B, 220 (2018) 222-233.

[28] P. Niu, L. Zhang, G. Liu, H.M. Cheng, Adv. Func. Mater. 22 (2012) 4763-4770

[29] A. Akhundi, E. I. García-López, G. Marcì, A. Habibi-Yangjeh, L. Palmisano, Res. Chem. Interm. 43 (2017) 5153-5168.

[30] M. Ilkaeva, I. Krivtsov, E. Bartashevich, S.A. Khainakov, J.R. García, E. Díaz, S. Ordóñez, Green Chem., 19 (2017) 4299-4304.

[31] Y. Kofuji, Y. Isobe, Y. Shiraishi, H. Sakamoto, S. Tanaka, S. Ichikawa, T. Hirai, J. Am. Chem. Soc., 138 (2016) 10019-10025.

[32] S. Malato Rodriguez, J. Blanco Galvez, M.I. Maldonado Rubio, P. Fernandez Ibañez, D. Alarcon Padilla, M. Collares Pereira, J. Farinha Mendes, J. Correia de Oliveira, Solar Energy 77 (2004) 513-524.

[33] J. Colina-Márquez, F. Machuca-Martínez, G. Li Puma, Env. Sci. Technol. 44 (2010) 51125120 .

[34] Y. Shiraishi, S. Kanazawa, Y. Sugano, D. Tsukamoto, H. Sakamoto, S. Ichikawa, T. Hirai, ACS Catal. 4 (2014) 774-780.

[35] F. Su, S.C. Mathew, G. Lipner, X.Fu, M. Antonietti, S. Blechert, X. Wang, J. Am. Chem. Soc. 132 (2010) 16299-16301.

[36] G. Palmisano, M. Addamo, V. Augugliaro, T. Caronna, A. Di Paola, E. García-López, V. Loddo, G. Marcì, L. Palmisano, M. Schiavello, Catal Today 122 (2007) 118-127. 